\title{
25 Research Square \\ Flavivirus Infection Induce a Golgi Stress Response in Vertebrate and Mosquito Cells
}

\section{Mercedes Viettri}

Center for Research and Advanced Studies (CINVESTAV-IPN) Mexico City

José L. Zambrano

Venezuelan Institute for Scientific Research (IVIC)

\section{Romel Rosales-Ramirez}

Center for Research and Advanced Studies (CINVESTAV-IPN) Mexico City

\section{Ana Lorena Gutierrez-Escolano}

Center for Research and Advanced Studies (CINVESTAV-IPN) Mexico City

Juan E Ludert ( $\square$ jludert@cinvestav.mx )

Center for Research and Advanced Studies (CINVESTAV-IPN) Mexico City

\section{Research Article}

Keywords: Flavivirus, dengue virus, Zika virus, dengue NS1, Golgi-stress response, TFE3

Posted Date: June 15th, 2021

DOI: https://doi.org/10.21203/rs.3.rs-603418/v1

License: (c) (i) This work is licensed under a Creative Commons Attribution 4.0 International License.

Read Full License

Version of Record: A version of this preprint was published at Scientific Reports on December 1st, 2021. See the published version at https://doi.org/10.1038/s41598-021-02929-1. 


\section{Abstract}

The stress of the Golgi apparatus is an autoregulatory mechanism that is induced to compensate for greater demand in the Golgi functions. Few examples of Golgi stress responses due to physiological stimuli are known. Furthermore, the impact on this organelle of viral infections that occupy the vesicular transport during replication is unknown. In this work, we evaluated if a Golgi stress response is triggered during dengue and Zika viruses replication, two flaviviruses whose replicative cycle is heavily involved with the Golgi complex, in vertebrate and mosquito cells. Using GM-130 as a Golgi marker, and treatment with monensin as a positive control for the induction of the Golgi stress response, a significant expansion of the Golgi cisternae was observed in BHK-21, Vero E6 and mosquito cells infected with either virus. Activation of the TFE3 pathway was observed in the infected cells as indicated by the translocation from the cytoplasm to the nucleus of TFE3. Of note, no sign of activation of the stress response was observed in CRFK cells infected with Feline Calicivirus (FCV), a virus released by cell lysis, not requiring vesicular transport. Finally, dilatation of the Golgi complex and translocation of TFE3 was observed in vertebrate cells expressing dengue and Zika viruses NS1, but not NS3. These results indicated that infections by dengue and Zika viruses induce a Golgi stress response in vertebrate and mosquito cells due to the increased demand on Golgi complex imposed by virion and NS1 processing and secretion.

\section{Introduction}

The endoplasmic reticulum (ER) and the Golgi apparatus are two tightly connected organelles that are key in the synthesis, processing and secretion of eukaryotic cells proteins. These organelles are bound by membranes that allow for the compartmentalization of specialized cellular functions. Upon an excessive, overwhelming demand of the functions of the ER or the Golgi apparatus, these organelles respond by a mechanism of homeostatic autoregulation termed stress responses, aimed to augment their functional capacity ${ }^{1-3}$.

Viral infections are inductors of stress in the infected cell. In particular, flavivirus replication processes leading to the production of progeny virions, interfere with a number of aspects of the cellular metabolism, triggering a homeostatic disequilibrium ${ }^{4,5}$. The flavivirus genome is a single stranded positive sense RNA molecule of approximately $10 \mathrm{~kb}$ in length, which encodes one large polyprotein that, after proteolytic processing by host and viral proteases, gives rise to the three structural proteins (capsid, Pr-M and envelope) and seven no structural proteins (NS1, NS2A, NS2B, NS3, NS4A, NS4B and NS5) that play essential roles in genome replication, polyprotein processing and virion assembly. Translation and replication of the flavivirus genome, as well as virion formation are all processes that occur in the ER and involve extensive ER membrane modifications ${ }^{6-9}$. Consequently, ER stress and the activation of the Unfolded-Protein-Response (UPR) have been well documented in vertebrate and mosquito cells infected with dengue (DENV) or Zika (ZIKV) viruses ${ }^{5,10-13}$.

After leaving the ER complex, immature virions enter the Golgi apparatus and following a classical secretory pathway, are transported to the plasma membrane to be released out of the cell by exocytosis. 
During their transit thru the Golgi complex, immature virions undergo glycan modifications in the envelope $(E)$ protein and proteolytic cleavage of the $\mathrm{pM}$ protein, by action of the Golgi resident protease furin, to become mature infectious virions ${ }^{14-16}$. In addition, DENV and ZIKV infected vertebrate and mosquito cells, also secrete the NS1 protein as a hexamer. In vertebrate cells, the NS1 protein is released following a classical secretory pathway using the Golgi apparatus, where the N-glycans attached to NS1 suffer further processing 5,6,8,17. All those secretory processes may impact and overwhelm the capacity of the Golgi.

The Golgi apparatus is an organelle with various functional zones, including the cis-Golgi network, cisGolgi, medial-Golgi, trans-Golgi, trans-Golgi network, separated by membranes and distinct cisterns, where diverse cellular functions such as glycosylation, sulfation, fucosylation and phosphorylation of secretory and membrane proteins is carried out ${ }^{2,3}$. In the cells that need a greater demand for the functions of Golgi, as could be the case of flaviviruses infected cells, the capacity of this organelle collapses. To compensate this functional demand a mechanism of autoregulation homeostatic is induced, which is termed Golgi stress response which involve several activation or response pathways ${ }^{1-3,18}$.

In this work, we explore if the high involvement of the Golgi complex during flavivirus replication results in the induction of the Golgi stress response. Our results indicate that DENV and ZIKV infection activates the Golgi stress response in both vertebrate and mosquito cell lines. Moreover, the sole expression of the NS1 protein can induce the stress response in vertebrate cells. Interestingly, the replication of feline calicivirus, a virus not involved with the Golgi apparatus, do not result in a stress response. The understanding of the activation and regulation of the Golgi stress response is still incomplete ${ }^{3}$; our results suggest that flavivirus infections can become an additional tool to study this phenomena.

\section{Results}

Flavivirus infection results in a Golgi stress response in both vertebrate and mosquito cells. The Golgi stress response is a consequence of an increased demand on the organelle functions. Given that flavivirus replication and NS1 and virion secretion are processes heavily dependent on the Golgi apparatus, we tested if a Golgi stress response is induced in cells infected with DENV or ZIKV. As a positive control, the $\mathrm{Na}^{+} / \mathrm{H}^{+}$ionophore monensin was used at non-toxic concentrations. Monensin is an agent that blocks glycosylation and vesicular transport of proteins and induce Golgi-stress response in treated cells ${ }^{3,19}$. Golgi expansion or fragmentation was visualized using GM-130 as a marker. In mock infected cells, GM-130 mark was observed a dotted staining near the nuclei of the cell, that correspond to the reporter Golgi subcellular localization (Figure. 1). Monensin treatment results in a fuzzy pattern of GM-130 staining throughout all the cytoplasm, indicating fragmentation and dispersion of the Golgi apparatus in treated cells (Fig. 1). A pattern like the one obtained with monensin treatment was observed in the Vero, BHK-21 and C6/36 cells infected with DENV and ZIKV at 48 hours post-infection (Fig. 1). These results suggest that DENV and ZIKV replication results in a Golgi stress response in vertebrate as well as mosquito cells. 
DENV and ZIKV infection in vertebrate cells results in the activation of the transcription factor E3 (TFE3) pathway. One of the pathways activated during the Golgi stress response is the TFE3 pathway. TFE3 located in the cytoplasm, is dephosphorylated and translocated to the cell nuclei upon activation of the Golgi stress response. To corroborate the activation of the stress response, vertebrate cells transfected with a recombinant form of TFE3 were infected with DENV and ZIKV. As shown in Fig. 2A, in untreated cells the mark corresponding to TFE3 is located mainly in the cytoplasm. However, monensin treatment or viral infections resulted in a significant translocation of TFE3 from the cytoplasm to the cell nuclei. In Vero-E6 cells, a statistically significant difference was observed in the percentage of TFE3 translocation between the mock condition $(27.8 \%)$ and cells treated with monensin $(95.2 \%)$ or cells infected with DENV (88.2\%) or ZIKV (83.9\%). A similar effect was observed in BHK 21 cells, with a percentage of TFE3 translocation of $21.7 \%$ in untreated cells, $96.8 \%$ in cells treated with monensin, $78 \%$ in DENV infected cells and $80.4 \%$ in ZIKV infected cells (Fig. 2B). These results indicate that the TFE3 pathway is activated in vertebrate cells infected with DENV and ZIKV. Since no expression of TFE3 was observed in transfected mosquito cells, C6/36 were not considered in this experiment.

FCV infection do not activate the Golgi stress response. FCV is a heavily lytic virus, whose replication cycle have little or no involvement with the Golgi apparatus ${ }^{20}$. Thus, to address if the observed activation of the Golgi stress response was specific for DENV and ZIKV infections, CRFK cells infected with FCV were analyzed for Golgi expansion or translocation of the TFE3 factor. GM-130 staining of mock infected CRFK cells was observed as a dotted mark near the nuclei. In addition, TFE3 was observed in the cytoplasm of untreated cells. No changes in the architecture of the Golgi complex or translocation of the TFE3 factor to the cell nuclei were observed in infected CRFK fixed at 5 and $7 \mathrm{hpi}$ (Fig. 3). Yet, CRFK cells can mount a Golgi stress response as indicated by the results obtained in monensin treated cells, where changes in the GM-130 mark and translocation of TFE3 were observed. These results suggest that demanding of Golgi functions during the virus replicative process of DENV and ZIKV, but not FCV, are necessary for activation of the Golgi stress response.

Expression of recombinant DENV NS1, but not NS3, results in activation of the Golgi stress response. The secretion of hexameric NS1 in vertebrate cells takes place following the classical secretory route and the Golgi apparatus. To test if the secretion of NS1 alone was capable of triggering the Golgi response, Vero and BHK-21 cells were transfected with a plasmid expressing a recombinant DENV NS1 protein, able to be secreted. Expression of the recombinant NS1 of DENV for 24 hours resulted in activation of the Golgi stress response in Vero-E6 and BHK-21 cells, as seen by the diffuse cytoplasmic GM-130 staining in both transfected cells. On the other hand, when the ER resident NS3 protease was expressed, no activation of the response was observed. (Fig. 4). These results suggest that the expression and transit of NS1 alone is sufficient to activate the Golgi stress response in vertebrate cells.

\section{Discussion}

The mosquito borne flaviviruses DENV and ZIKV are etiologic agents of important public health diseases affecting tropical and subtropical countries around the globe ${ }^{21}$. The replicative cycle of these and other 
flavivirus takes place in close contact with membrane bound organelles which are directly or indirectly affected by the viral replicative process. Extensive ER membrane modifications and induction of an UPR, changes in the shape of mitochondria, and even nuclear pore degradation have all been reported in DENV infected cells ${ }^{13,22,23}$. Yet, and despite that the processing and secretion of hexameric NS1 and mature virions in vertebrate cells, as well as the processing and secretion of mature virions in mosquito cells, are all processes that take place in the Golgi apparatus ${ }^{17}$, little is known regarding the response of the Golgi system to flavivirus infections. In this work, evidence obtained in three different cell lines, from vertebrate and mosquito origin, is presented indicating that DENV and ZIKV infections triggers the Golgi stress response and the activation of the TFE3 pathway in infected vertebrate cells. If other mosquito borne flaviviruses not included in this study, such as JEV or WNV, or even the HCV induce a Golgi stress response in infected cells remains to be determined, but given the heavy involvement of all flaviviruses with the Golgi apparatus ${ }^{7}$, this is expected to be the case. Interestingly, the activation of the Golgi stress response seems not to be a generalized phenomenon of viral infections as suggested by the observation that FCV does not trigger these responses in CRFK infected cells, although these cells possess all the molecular mechanisms necessary to go into Golgi stress.

In their transit to the extracellular space, immature DENV and ZIKV virions enter the Golgi apparatus where the E protein suffers trimming of the $\mathrm{N}$-glycan residues ${ }^{24}$ and later the prM protein is proteolytically processed by the Golgi resident acidic protease furin to finally form mature virions ${ }^{15}$. In addition, in vertebrate cells, the secretion of NS1 protein from infected cells utilizes the entire Golgi system, where the two $\mathrm{N}$-glycan residues attached to NS1 undergo further processing ${ }^{24}$. Moreover, the NS1 located on the plasma membrane is GPI-anchored protein, another function that take place in the Golgi cisternae ${ }^{25}$. More recently, multiple components of the Golgi apparatus were found in the interactome of NS1 in vertebrate cells ${ }^{26}$. In this work, we demonstrate that activation of the Golgi stress response occurs also in cells expressing NS1 by itself. So, along with vesicle transport, flavivirus proteins such as E, pr-M and NS1 suffer several post-transcriptional modifications in their transit over the Golgi cisternae. The TFE3 pathway activation ends in the expression of genes involved in augmenting the functions of the Golgi apparatus in general, including Golgi structure, N-glycosylation functions and vesicular transport, rather than a specific function ${ }^{3}$. Thus, the activation of the TFE3 pathway in DENV and ZIKV infected cells is in line with the import and multiple roles played by the Golgi complex during flavivirus replication. Other pathways also activated during the Golgi stress response, such as the CREB3 and the HSP47 pathways conduct to the activation of pro-apoptotic and anti-apoptotic genes ${ }^{3}$. The infection of mouse endothelial cells by HSV-1 causes GM-130 degradation and Golgi apparatus fragmentation that finally leads to cell apoptosis ${ }^{27}$. Both, activation and suppression of apoptosis have been reported in DENV infected cells ${ }^{28,29}$. If the CREB3 or the HSP47 pathways are activated during DENV and ZIKV replication is unknown.

The UPR triggered during flavivirus replication have been linked with mechanisms favoring viral replication such as modulation of innate immunity and induction of autophagy ${ }^{10,28,30}$. The significance 
of the activation of the Golgi stress response during flavivirus replicative cycle is unclear, but it is reasonable to speculate that augmenting the capacity of the Golgi apparatus will favor the secretion of NS1 and mature virions. Consequently, a better understanding of the interplay between DENV and ZIKV proteins and the Golgi apparatus may help to identify antiviral targets common to vertebrate and mosquito cells.

Finally, we like to remark that several molecular aspects of the Golgi stress response, as for example, the sensor molecule for the activation of the TFE3 pathway, are still unknown. Currently, the TFE3 pathway is activated mainly using pharmacological treatments, such as monensin and nigericin ${ }^{3}$. The results here presented suggest that infections with DENV and ZIKV, or expression of recombinant NS1 may become additional experimental tools in the study of the Golgi apparatus and its stress response. Numerous are the examples of how the study of viruses had been rewarding to study the biology of cells.

\section{Materials And Methods}

Cell cultures. C6/36 mosquito cells from Aedes albopictus (ATCC $\circledast$ CRL-1660 19 ) were grown at $28^{\circ} \mathrm{C}$ in Eagle's Minimum Essential Medium (EMEM) (ATCC® 30-2003 ${ }^{\text {TM }}$ ), supplemented with $5 \%$ fetal bovine serum (FBS) and $100 \mathrm{U} / \mathrm{ml}$ penicillin-streptomycin. Baby hamster kidney cells (BHK-21, ATCC $\AA$ CCL-10 $0^{\text {TM }}$ ) were grown at $37^{\circ} \mathrm{C}$ and cultured in Eagle's Minimum Essential Medium (EMEM, ATCC $®$ ) supplemented with $5 \% \mathrm{FBS}$ and $100 \mathrm{U} / \mathrm{ml}$ penicillin-streptomycin. Monkey kidney epithelial cells Vero E6 (ATCC® CRL$1586^{\mathrm{TM}}$ ) were grown at $37^{\circ} \mathrm{C}$ and cultured in Eagle's Minimum Essential Medium (EMEM, ATCC ${ }^{\circ}$ ) supplemented with $10 \%$ FBS and $100 \mathrm{U} / \mathrm{ml}$ penicillin-streptomycin. Crandell Resse feline kidney (CRFK, ATCC $\circledast$ CCL-94) cells were grown at $37^{\circ} \mathrm{C}$ in Eagle's Minimal Essential Medium with Earle's balanced salt solution, $2 \mathrm{mM}$ l-glutamine, $1.0 \mathrm{mM}$ sodium pyruvate, $0.1 \mathrm{mM}$ nonessential amino acids, $1.5 \mathrm{~g} / \mathrm{l}$ sodium bicarbonate, supplemented with $10 \%$ bovine fetal serum, $5000 \mathrm{U}$ of penicillin, and $5 \mu \mathrm{g} / \mathrm{ml}$ of streptomycin. All cell lines were grown in a $5 \% \mathrm{CO}_{2}$ incubator.

Virus strains. DENV serotype 2, strain New Guinea and a Mexican isolate from A. aegypti of ZIKV, Asian genotype, were generously provided by M.Sc. Mauricio Vázquez, (Laboratorio de Arbovirus y Virus Hemorrágicos. Instituto de Diagnóstico y Referencia Epidemiológicos. InDRE, Mexico City) and propagated in C6/36 cells. DENV and ZIKV titers were determined by focus forming assays ${ }^{31}$. The feline calicivirus (FCV, strain F9) was propagated and titrated by plaque forming units assay in CRFK cells ${ }^{32}$.

Drug treatment. Monensin disodium salt (M5273, Sigma-Aldrich $\left.{ }^{\circledR}\right)$ was dissolved in ethanol to a concentration of $10 \mathrm{mM}$. Monensin is an inductor of the Golgi stress response and was used as positive control. Cells were treated with Monensin at a final concentration of $5 \mu \mathrm{M}$ in EMEM supplemented with $5 \%$ FBS, for $24 \mathrm{~h}$. The conditions for non-toxic treatment of the different cell lines with Monensin were determined previously, using cell viability MTT assays, according to the manufactures' instructions (V13164, Themofisher). 
Virus infections. Confluent cell monolayers grown in 24-well plates $\left(2 \times 10^{5}\right.$ cells per well) were infected for 2 hours, using a $\mathrm{MOI}=3$ for DENV and ZIKV, and a $\mathrm{MOI}=5$ for FCV. After infection, the monolayers were washed three times with PBS to remove unabsorbed viruses, and infections allowed to proceed for $48 \mathrm{~h}$ for DENV or ZIKV, and 5 and $7 \mathrm{~h}$ for FCV. Afterward, cells were washed, fixed and stained for immunofluorescence.

Plasmids transfection. Plasmids expressing recombinant DENV-NS1 and DENV-NS3 were kindly donated by Dr. Ana Fernández-Sesma (Icahn School of Medicine at Mount Sinai, New York). Recombinant plasmids expressing the transcriptional factor E3 (pTFE3-Myc) were kindly donated by Dr. Hiderou Yoshida (University of Hyogo, Japan). Plasmids were transfected into confluent monolayer of cells grown in 24-well plates using lipofectamine reagent Lipofectamine ${ }^{\mathrm{TM}} 2000$ (11668019, Invitrogen); each well was transfected with $1 \mu \mathrm{g}$ of plasmid DNA and $2 \mu \mathrm{L}$ of Lipofectamine in a final volume of $0,25 \mathrm{ml}$ of EMEM w/o FBS. After $5 \mathrm{~h}$ of transfection, $0.25 \mathrm{ml}$ of EMEM supplemented with $10 \%$ FBS were added per well. Cells transfected for DENV-NS1 and DENV-NS3 were fixed and stained for immunofluorescence at $24 \mathrm{~h}$ post transfection. Cells transfected with pTFE3-Myc were infected with DENV or ZIKV $24 \mathrm{~h}$ after transfection and the infection allowed to proceed for additional $48 \mathrm{hrs}$ before fixing and staining for immunofluorescence. In addition, cells transfected with pTFE3-Myc were treated with monensin for $24 \mathrm{~h}$, fixed and stained for immunofluorescence.

Confocal microscopy. Confluent cell monolayers, grown in 24-well plates containing glass coverslips, were infected or transfected as described above. At the indicated times, cells were washed once with PBS, fixed in paraformaldehyde $4 \%$ for 10 minutes and permeabilized with $0.1 \%$ Triton X-100 for 10 minutes at room temperature. Cells were stained for DENV-NS1 using Mab2B7, for DENV-NS3 using Mab 1ED8 (both Mabs a kind gift of Dr. Eva Harris, UC Berleley), for FCV-VP1 (SC65625, Santa Cruz Biotechnology), the Golgi complex was visualized using a commercial anti-GM130 antibody (GTX130351, GeneTex), and the TFE3 using a commercial anti-Myc antibody (GTX30518, GeneTex), as primary antibodies. As secondary antibodies, anti-mouse Alexa-488, anti-rabbit Alexa-568 and anti-goat Alexa-568 conjugated (donkey preadsorbed, secondary antibodies, Abcam) were used at 1:800 dilution in PBS. Nuclei were counter stained with DAPI (D9542, Sigma-Aldrich $\left.{ }^{\circledR}\right)$. Coverslips were mounted in Fluoroshield ${ }^{\text {TM }}$ with DAPI (F6057, SigmaAldrich $\circledast$ ). The slides were examined using a Zeiss LSM 700 confocal microscope.

Translocation of recombinant transcriptional factor E3. Percentage of translocation of TFE3 from the cytoplasm to the nucleus was calculated through the comparison of the median fluorescence intensity (MFI) in the nucleus versus the cytoplasm (MFI nucleus area/MFI cytoplasm area $x 100$ ) in each of the experimental conditions. At least 10 cells per condition were examined and the results analyzed using test one-way ANOVA. The images were processed with Zen Blue edition 2.6 software and the results were plotted with the GraphPAD Prism version 8.01 .

\section{Declarations}


ACKNOWLEDGEMENTS. Authors like to thank Carlos Vasquez for technical assistant in the confocal microscopy. This work was partially supported CONACYT grant CB-254461 and IGCEB grant CRP/MEX1702 to JEL. JLZR was a visiting professor at the Department of Infectomics and Molecular Pathogenesis, CINVESTAV, Mexico supported by a scholarship of the UNU-BIOLAC program (Contract CTC284). Authors declared no conflict of interest.

AUTHORS CONTRIBUTION. MV, RRR and JEL conceived and design the study. MV, JLZ and RRR performed the experiments. ALGE and JEL contributed reagents, materials and analytical tools. MV wrote the first draft. JEL wrote the final version of the manuscript. All authors reviewed the final manuscript.

\section{References}

1. Yoshida, H. ER stress response, peroxisome proliferation, mitochondrial unfolded protein response and golgi stress response. IUBMB Life 61, 871-879 (2009).

2. Sasaki, K. \& Yoshida, H. Organelle autoregulation - Stress responses in the ER, Golgi, mitochondria and lysosome. J. Biochem. 157, 185-195 (2015).

3. Sasaki, K. \& Yoshida, H. Golgi stress response and organelle zones. Fed. Eropean Biochem. Soc. Lett. 593, 2330-2340 (2019).

4. Fernandez-Garcia, M. D., Mazzon, M., Jacobs, M. \& Amara, A. Pathogenesis of Flavivirus Infections: Using and Abusing the Host Cell. Cell Host Microbe 5, 318-328 (2009).

5. Valadão, A. L. C., Aguiar, R. S. \& de Arruda, L. B. Interplay between inflammation and cellular stress triggered by Flaviviridae viruses. Front. Microbiol. 7, 1-19 (2016).

6. Apte-Sengupta, S., Sirohi, D. \& Kuhn, R. J. Coupling of Replication and Assembly in Flaviviruses Swapna. Curr Opin Virol 100, 130-134 (2014).

7. Chatel-Chaix, L. \& Bartenschlager, R. Dengue Virus- and Hepatitis C Virus-Induced Replication and Assembly Compartments: the Enemy Inside--Caught in the Web. J. Virol. 88, 5907-5911 (2014).

8. Garcia-Blanco, M. A., Vasudevan, S. G., Bradrick, S. S. \& Nicchitta, C. Flavivirus RNA transactions from viral entry to genome replication. Antiviral Research (2016).

9. Sager, G., Gabaglio, S., Sztul, E. \& Belov, G. A. Role of host cell secretory machinery in zika virus life cycle. Viruses 10, 1-18 (2018).

10. Lee, Y.-R. et al. Dengue virus-induced ER stress is required for autophagy activation, viral replication, and pathogenesis both in vitro and in vivo OPEN. Scientific 8, 1-14 (2018).

11. Sepúlveda-Salinas, K. J. \& Ramos-Castañeda, J. Participation of dengue virus NS4B protein in the modulation of immune effectors dependent on ER stress in insect cells. Cell Stress Chaperones 22, 799- 
810 (2017).

12. Tan, Z. et al. ZIKV infection activates the IRE1-XBP1 and ATF6 pathways of unfolded protein response in neural cells. J. Neuroinflammation 15, 1-16 (2018).

13. Yu, C.-Y., Hsu, Y.-W., Liao, C.-L. \& Lin, Y.-L. Flavivirus Infection Activates the XBP1 Pathway of the Unfolded Protein Response To Cope with Endoplasmic Reticulum Stress. J. Virol. 80, 11868-11880 (2006).

14. Stadler, K., Allison, S. L., Schalich, J. \& Heinz, F. X. Proteolytic activation of tick-borne encephalitis virus by furin. J. Virol. 71, 8475-8481 (1997).

15. Mukherjee, S. et al. Enhancing dengue virus maturation using a stable furin over-expressing cell line. Virology 497, 33-40 (2016).

16. Roby, J. A., Setoh, Y. X., Hall, R. A. \& Khromykh, A. A. Post-translational regulation and modifications of flavivirus structural proteins. J. Gen. Virol. 96, 1551-1569 (2015).

17. Alcalá, A. C., Palomares, L. A. \& Ludert, J. E. Secretion of Nonstructural Protein 1 of Dengue Virus from Infected Mosquito Cells: Facts and Speculations. J. Virol. 92, 1-9 (2018).

18. Taniguchi, M. \& Yoshida, H. TFE3, HSP47, and CREB3 pathways of the mammalian golgi stress response. Cell Struct. Funct. 42, 27-36 (2017).

19. Dinter, A. \& Berger, E. G. Golgi-disturbing agents. Histochem. Cell Biol. 109, 571-590 (1998).

20. Radford, A. D., Coyne, K. P., Dawson, S., Porter, C. J. \& Gaskell, R. M. Review article Feline calicivirus. Vet. Res. 38, 319-335 (2007).

21. Higuera, A. \& Ramírez, J. D. Molecular epidemiology of dengue, yellow fever, Zika and Chikungunya arboviruses: An update. Acta Trop. 190, 99-111 (2019).

22. Chatel-Chaix, L. et al. Dengue Virus Perturbs Mitochondrial Morphodynamics to Dampen Innate Immune Responses. Cell Host Microbe 20, 342-356 (2016).

23. de Jesús-González, L. A. et al. The nuclear pore complex: A target for NS3 protease of dengue and Zika viruses. Viruses 12, (2020).

24. Yap, S. S. L., Nguyen-Khuong, T., Rudd, P. M. \& Alonso, S. Dengue virus glycosylation: What do we know? Front. Microbiol. 8, 1-16 (2017).

25. Jacobs, M. G., Robinson, P. J., Bletchly, C., Mackenzie, J. M. \& Young, P. R. Dengue virus nonstructural protein 1 is expressed in a glycosyl-phosphatidylinositol-linked form that is capable of signal transduction. FASEB J. 14, 1603-1610 (2000). 
26. Hafirassou, M. L. et al. A Global Interactome Map of the Dengue Virus NS1 Identifies Virus Restriction and Dependency Host Factors. Cell Rep. 21, 3900-3913 (2017).

27. He, Q. et al. Herpes Simplex Virus 1-Induced Blood-Brain Barrier Damage Involves Apoptosis Associated With GM130-Mediated Golgi Stress. Front. Mol. Neurosci. 13, 1-16 (2020).

28. Perera, N., Miller, J. L. \& Zitzmann, N. The role of the unfolded protein response in dengue virus pathogenesis. Cell. Microbiol. 19, 1-9 (2017).

29. Okamoto, T. et al. Regulation of apoptosis during flavivirus infection. Viruses 9, 1-12 (2017).

30. Sepúlveda-Salinas, K. J. \& Ramos-Castañeda, J. Participation of dengue virus NS4B protein in the modulation of immune effectors dependent on ER stress in insect cells. Cell Stress Chaperones 22, 799810 (2017).

31. Rosales Ramirez, R. \& Ludert, J. E. The Dengue Virus Nonstructural Protein 1 (NS1) Is Secreted from Mosquito Cells in Association with the Intracellular Cholesterol Transporter Chaperone Caveolin Complex. J. Virol. 93, 1-25 (2019).

32. Escobar-Herrera, J., Medina-Ramírez, F. J. \& Gutiérrez-Escolano, A. L. A carboxymethyl-cellulose plaque assay for feline calicivirus. J. Virol. Methods 146, 393-396 (2007).

\section{Figures}




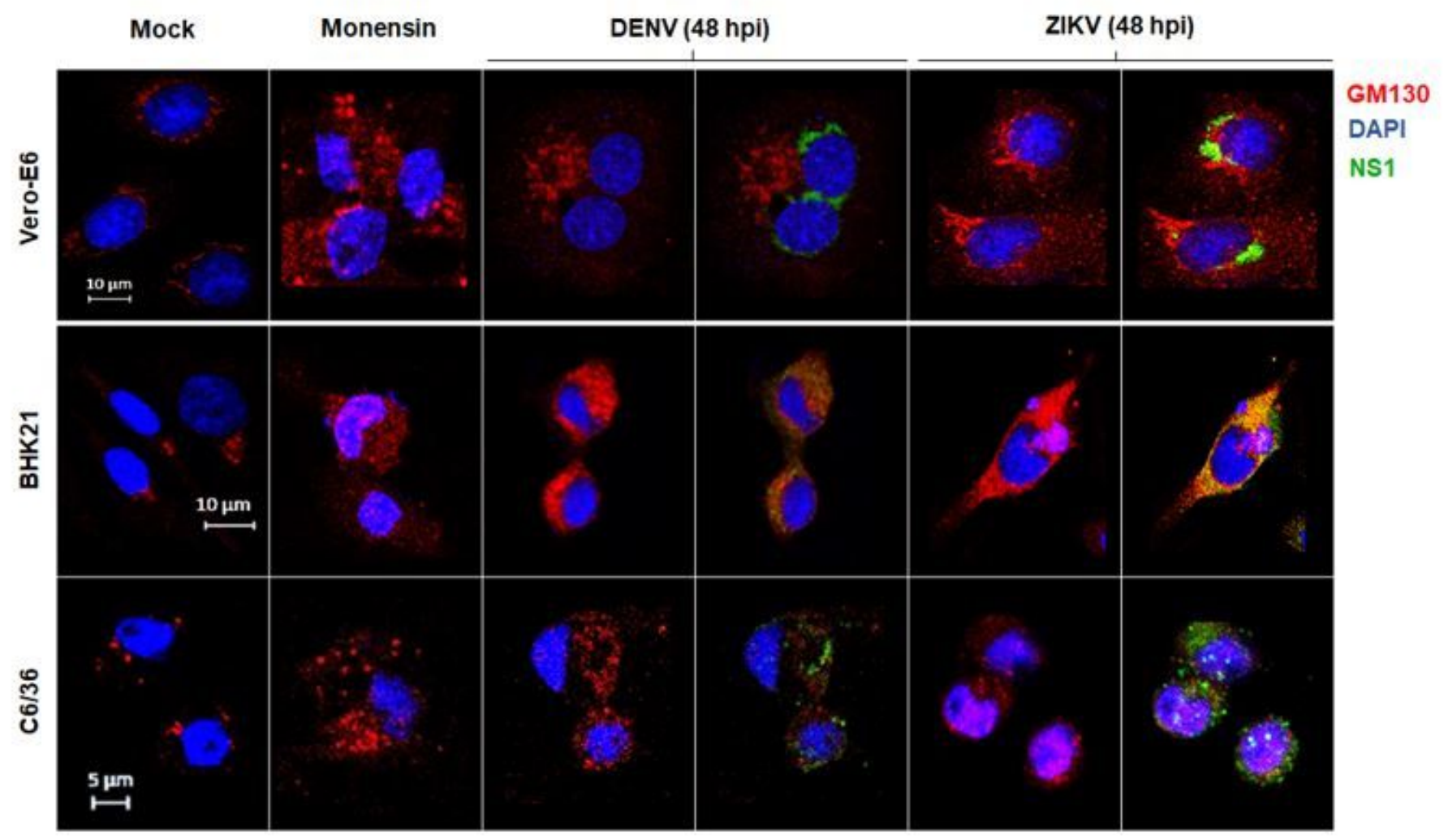

\section{Figure 1}

Golgi stress response due to flavivirus infection in vertebrate and mosquito cells. Cells were either treated with monensin and fixed at $24 \mathrm{~h}$ or infected with DENV and ZIKV at MOI= 3 and fixed at $48 \mathrm{hpi}$. Cells were probed against flavivirus NS1 (green), and GM130 (cis-Golgi, red). Nuclei were counter stained with DAPI (blue). Merged fluorescent images of DAPI, red and green channels are shown. The images were analyzed using a Zeiss LSM 700 confocal microscope with laser sections: $0.45 \mu \mathrm{m}$. Images from one out of least 3 independent experiments are shown. 

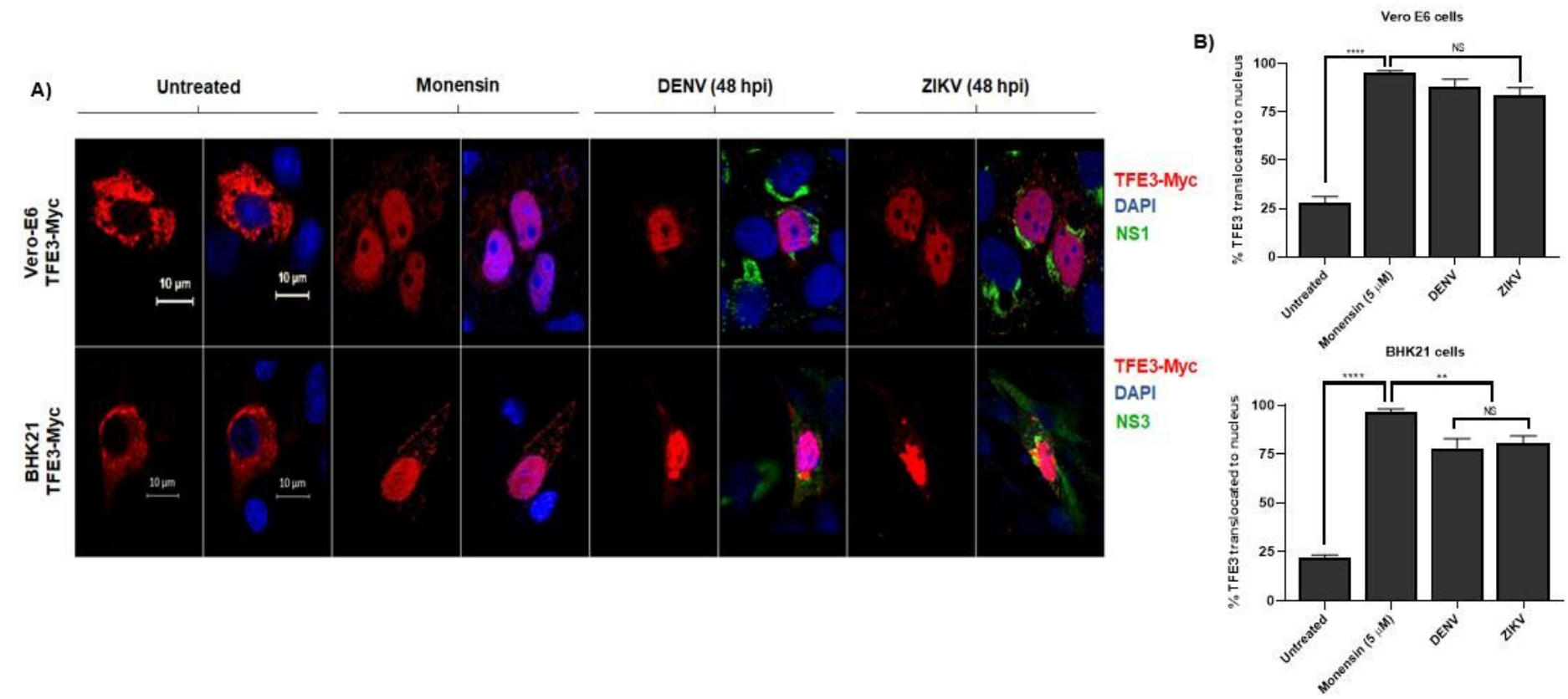

\section{Figure 2}

Activation of TFE3 pathway due flavivirus infection in vertebrate cells. A) Cells lines Vero-E6 and BHK21 were transfected with $1 \mu \mathrm{g}$ of plasmid DNA of transcriptional factor TFE3 (TEF3-Myc). Twenty-four $\mathrm{h}$ after transfection, cells were treated with monensin and fixed $24 \mathrm{~h}$ after treatement or infected with DENV and ZIKV and fixed 48 hpi. Cells were probed against flavivirus NS1 (green), and Myc (TFE3, red). Nuclei were counter stained with DAPI (blue). Merged fluorescent images of DAPI, red and green channels are shown. The images were analyzed using a LSM 700 confocal microscope. The images were analyzed using a LSM 700 confocal microscope with laser sections: $0.45 \mu \mathrm{m}$. Images from one out of least 3 independent experiments are shown. B) Percentage of TFE3 translocated from the cytoplasm to nucleus in both cells lines were measured through images analysis with Zen Blue edition 2.6 software and the results were plotted using GraphPAD Prism version 8.01. * $p \leq 0.05$.
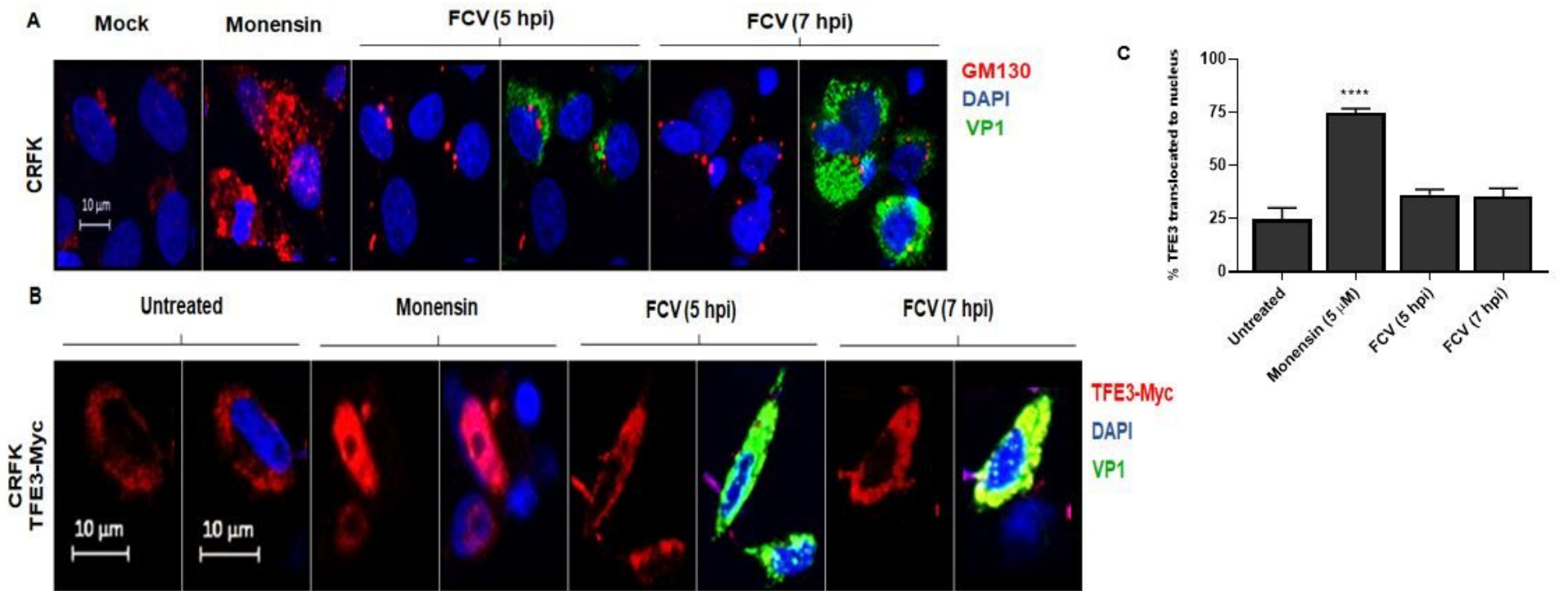

TFE3-Myc

DAPI

VP1 


\section{Figure 3}

CRFK cells infected with FCV showed no evidence of Golgi stress or activation of the TFE3 pathway. A) CRFK cells were infected with FCV and fixed at 5 and $7 \mathrm{hpi}$. The cells were probed against capsid protein VP1 (green), and GM130 (cis-Golgi, red). Nuclei were counter stained with DAPI (blue). B) CRFK cells were transfected with $1 \mu \mathrm{g}$ of plasmid DNA of transcriptional factor TFE3 (pTEF3-Myc), then infected with FCV at $\mathrm{MOI}=5$ and fixed at 5 and $7 \mathrm{hpi}$. Cells were probed against capsid protein VP1 (green), Myc (TFE3, red). Nuclei were counter stained with DAPI (blue). Cells were analyzed using a Zeiss LSM 700 confocal microscope with laser sections: $0.45 \mu \mathrm{m}$. C) Percentage of TFE3 translocated from the cytoplasm to nucleus were measured through images analysis with Zen Blue edition 2.6 software and the results were plotted using GraphPAD Prism version 8.01. * $p \leq 0.05$.

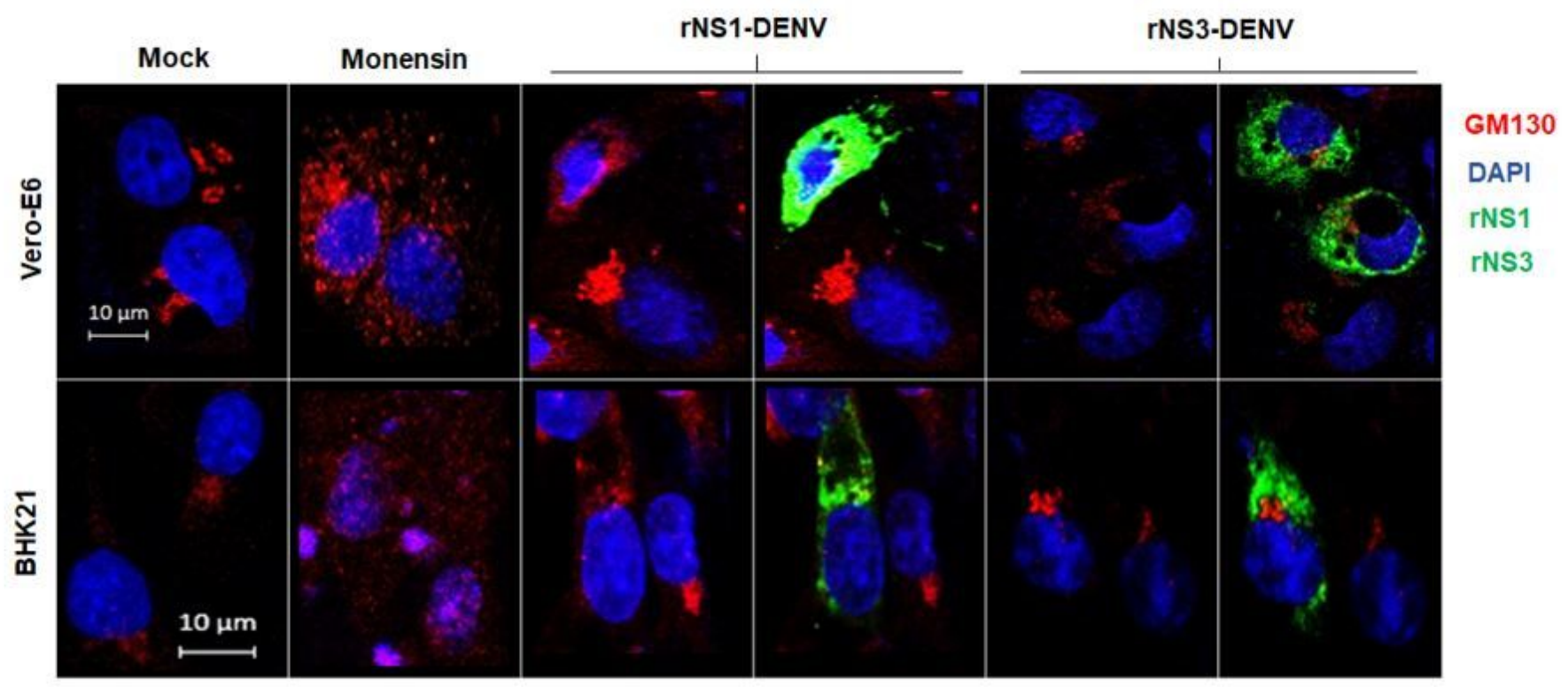

\section{Figure 4}

Golgi stress response in vertebrate cells due to expression of recombinant non-structural protein 1 of dengue virus. Cells were transfected with $1 \mu \mathrm{g}$ of plasmid DNA of DENV-NS1 and DENV-NS3 or treated with monensin as a positive control and fixed after 24 hours. Cells were probed against flavivirus NS1 and NS3 (green), GM130 (cis-Golgi, red). Nuclei were counter stained with DAPI (blue). Merged fluorescent images of DAPI, red and green channels are shown. The images were analyzed using a Zeiss LSM 700 confocal microscope with laser sections: $0.45 \mu \mathrm{m}$. Images from one out of least 3 independent experiments are shown. 